Cite this: Phys. Chem. Chem. Phys., 2013,

\title{
Process and reactor design for biophotolytic hydrogen production
}

15, 10783

Received 2nd May 2013,

Accepted 2nd May 2013

DOI: $10.1039 / \mathrm{c} 3 \mathrm{cp} 51866 \mathrm{c}$

www.rsc.org/pccp

\author{
Bojan Tamburic, Pongsathorn Dechatiwongse, Fessehaye W. Zemichael, \\ Geoffrey C. Maitland and Klaus Hellgardt*
}

\begin{abstract}
The green alga Chlamydomonas reinhardtii has the ability to produce molecular hydrogen $\left(\mathrm{H}_{2}\right)$, a clean and renewable fuel, through the biophotolysis of water under sulphur-deprived anaerobic conditions. The aim of this study was to advance the development of a practical and scalable biophotolytic $\mathrm{H}_{2}$ production process. Experiments were carried out using a purpose-built flat-plate photobioreactor, designed to facilitate green algal $\mathrm{H}_{2}$ production at the laboratory scale and equipped with a membrane-inlet mass spectrometry system to accurately measure $\mathrm{H}_{2}$ production rates in real time. The nutrient control method of sulphur deprivation was used to achieve spontaneous $\mathrm{H}_{2}$ production following algal growth. Sulphur dilution and sulphur feed techniques were used to extend algal lifetime in order to increase the duration of $\mathrm{H}_{2}$ production. The sulphur dilution technique proved effective at encouraging cyclic $\mathrm{H}_{2}$ production, resulting in alternating Chlamydomonas reinhardtii recovery and $\mathrm{H}_{2}$ production stages. The sulphur feed technique enabled photobioreactor operation in chemostat mode, resulting in a small improvement in $\mathrm{H}_{2}$ production duration. A conceptual design for a large-scale photobioreactor was proposed based on these experimental results. This photobioreactor has the capacity to enable continuous and economical $\mathrm{H}_{2}$ and biomass production using green algae. The success of these complementary approaches demonstrate that engineering advances can lead to improvements in the scalability and affordability of biophotolytic $\mathrm{H}_{2}$ production, giving increased confidence that $\mathrm{H}_{2}$ can fulfil its potential as a sustainable fuel of the future.
\end{abstract}

\section{Introduction}

\subsection{Sustainability through photosynthesis}

The world is facing serious environmental challenges at an unprecedented scale. Uncontrolled anthropogenic emissions of carbon dioxide are responsible for increasing the global temperature and acidifying the oceans. ${ }^{1}$ These changes have already resulted in reduced biodiversity ${ }^{2}$ and, unless addressed effectively, may pose a serious threat to our existence as a species. Anthropogenic carbon dioxide emissions are dominated by the combustion of fossil fuels for energy, implying that climate change is essentially an energy problem. Fossil fuels are not intrinsically dirty or unnatural, as many environmental groups would have us believe. ${ }^{3}$ They are the remnants of ancient microorganisms, which captured solar energy by photosynthesis and stored it within their biomass. However, the combustion of fossil fuels is not sustainable because there is a huge temporal discrepancy between the rapid consumption rate and the sedate geochemical production rate.

Department of Chemical Engineering, Imperial College London, UK.

E-mail: k.hellgardt@imperial.ac.uk
Our addiction to fossil fuels can be overcome by exploiting photosynthetic processes in real-time to generate solar fuels independently of the fossil fuel route. ${ }^{4}$ Green algae are a major contributor to the photosynthetic capacity of the biosphere. ${ }^{5}$ Often referred to as pond slime, they actually exhibit vast and complex biodiversity. They have adapted to living in the soil, in fresh water and in salt water, and they consequently inhabit the entire globe. Green algae are therefore affordable, available and flexible; they are an excellent target organism for photosynthetic biofuel production. Green algae are capable of producing multiple fuels, including biodiesel, bioethanol and biochar. ${ }^{6}$ From a sustainability perspective, the most exciting prospect is their ability to produce molecular hydrogen. $\mathrm{H}_{2}$ is the cleanest possible fuel since its combustion products are only heat energy and pure water. It has the highest energy density per unit mass of any fuel and it can be utilised efficiently in a proton exchange membrane fuel cell. The challenge of sustainable $\mathrm{H}_{2}$ production can be addressed using green algae. Green algae, such as the model organism Chlamydomonas reinhardtii (C. reinhardtii), produce $\mathrm{H}_{2}$ via the process of biophotolysis, which involves the splitting of water by photosynthesis to produce oxygen molecules, protons 
and electrons, and the subsequent recombination of these protons and electrons by the hydrogenase enzyme to produce molecular $\mathrm{H}_{2}{ }^{7}$ Biophotolytic hydrogen production has been demonstrated under anaerobic conditions at the laboratory scale. ${ }^{8}$

\subsection{Green algal biophotolysis}

C. reinhardtii is capable of producing $\mathrm{H}_{2}$ under anaerobic conditions by biophotolysis. Light energy is captured by lightharvesting proteins of the Photosystem II (PSII) reaction centre and used to split water into oxygen molecules, protons and electrons. These photosynthetic electrons are transported via a series of redox reactions to the iron-sulphur protein ferredoxin, an important biochemical electron donor. During photosynthetic growth, ferredoxin donates its electrons to NADPH (reduced nicotinamide adenine dinucleotide phosphate), the reductant of the Calvin cycle, which results in the fixation of carbon dioxide to starch, a prerequisite for all further biosynthesis. ${ }^{7}$ Under anaerobic conditions, ferredoxin instead donates its electrons to the [FeFe]-hydrogenase enzyme. The hydrogenase enzyme catalyses the process of proton-electron recombination to produce $\mathrm{H}_{2}$. In addition to the photosynthetic pathway, the hydrogenase enzyme also receives electrons from the catabolic breakup of starch and from the fermentation of pyruvate. ${ }^{9}$ The hydrogenase enzyme is extremely oxygen-sensitive, yet the algae constantly produce oxygen by photosynthesis; this presents a major challenge for the induction of $\mathrm{H}_{2}$ production. The solution is to remove the oxygen as it is being produced, creating an anaerobic environment. This can be achieved by purging the $C$. reinhardtii culture with an inert gas, by using an oxygen scavenger, or by subjecting the algae to a period of dark incubation or to a low light intensity. ${ }^{10}$ However, the most successful method for inducing $\mathrm{H}_{2}$ production has been twostage biophotolysis by sulphur-deprivation. ${ }^{8}$ Sulphur-deprivation reversibly reduces the activity of PSII without affecting the rate of respiration. The rate of photosynthetic oxygen production drops below the level of respirative oxygen consumption, resulting in a net consumption of oxygen. The algae metabolically bring themselves into a state of anaerobic $\mathrm{H}_{2}$ production. ${ }^{8}$ Anaerobic C. reinhardtii cells use ATP (adenosine triphosphate) as their primary energy source, but this does not provide sufficient energy to satisfy their long-term metabolic requirements. Anaerobic conditions therefore result in gradual cell death and the $C$. reinhardtii culture can only survive for 5-7 days. ${ }^{11}$

\subsection{Challenges to biophotolytic $\mathrm{H}_{2}$ production}

Green algal $\mathrm{H}_{2}$ has the potential to be a sustainable fuel of the future, if a number of technological challenges can be overcome. These obstacles include the low photochemical efficiency of biophotolysis, ${ }^{12}$ the short duration of the process, ${ }^{13}$ and the high cost of commercial photobioreactors. ${ }^{14}$ The crucial question that has to be answered is whether green algal $\mathrm{H}_{2}$ production can be scalable and affordable. Experimental techniques that enable biohydrogen production over long time periods, at large scales, and under ambient conditions, need to be developed. The aim of the Solar Hydrogen Project at Imperial College
London is to address some of these challenges. The objectives of this study are to:

- present a custom-built photobioreactor capable of facilitating green algal biophotolysis at the laboratory scale,

- introduce the practical and economical nutrient control method of $\mathrm{H}_{2}$ production, which enables spontaneous $\mathrm{H}_{2}$ production following algal growth,

- develop sulphur re-insertion techniques, sulphur dilution and sulphur feed, aimed at extending $\mathrm{H}_{2}$ production duration, and

- devise a photobioreactor concept for long-term biophotolytic $\mathrm{H}_{2}$ production at large scales.

\section{Experimental methods}

\subsection{Algae and algal growth media}

All experiments use cc124, a common laboratory wild-type strain of $C$. reinhardtii (kindly provided by Professor Peter J. Nixon, Imperial College London). Stock C. reinhardtii cultures were grown in the Tris-acetate phosphate (TAP) growth medium, prepared according to the classic recipe devised by Gorman and Levine. ${ }^{15}$ The TAP medium provides all the necessary nutrients for algal growth (carbon, nitrogen, sulphur, phosphorus and trace metals) but it also contains expensive and impractical ingredients such as acetic acid, ammonia and Tris buffer. ${ }^{16}$ Optimising the use of acetate is important for limiting biohydrogen production costs, particularly in large-scale systems. ${ }^{17}$ Since sulphurdeprivation induces the onset of anaerobic $\mathrm{H}_{2}$ production, controlling sulphate levels is an intuitive way of controlling $\mathrm{H}_{2}$ production. The classical TAP medium is overloaded with all key nutrients except for acetate, which normally runs out in the first three days of algal growth. ${ }^{18}$ Two variations of the TAP medium were used in experiments: TAP-S and $\mathrm{TAP}_{12}{ }^{19}$ The TAP$\mathrm{S}$ medium was prepared by replacing sulphate salts with their chloride derivatives; it provides a sulphur-deprived environment, which is necessary for the induction of $\mathrm{H}_{2}$ production. The $\mathrm{TAP}_{12}$ medium was designed to facilitate nutrient-controlled $\mathrm{H}_{2}$ production in the flat-plate PBR at a light intensity of $12 \mathrm{~W} \mathrm{~m}^{-2} \cdot{ }^{19}$

\subsection{Flat-plate photobioreactor inventory}

A novel flat-plate photobioreactor (PBR) has been designed and constructed (Paul Crudge, Imperial College London) specifically to facilitate biophotolytic $\mathrm{H}_{2}$ production at the laboratory scale. ${ }^{20}$ All experiments described in this study were carried out in the flat-plate PBR. The light intensity incident on the algal culture was controlled at $12 \mathrm{~W} \mathrm{~m}^{-2}\left(60 \mu \mathrm{E} \mathrm{m}^{-2} \mathrm{~s}^{-1}\right)$, and the temperature was controlled at $25{ }^{\circ} \mathrm{C}$ for all experiments. The optical density (OD) and dissolved oxygen content $\left(\mathrm{pO}_{2}\right)$ of the algal culture were measured in situ, using a photodiode operating with an absorption bandwidth of 650-700 nm (RS Components) and the SZ10T galvanic $\mathrm{pO}_{2}$ electrode (Consort), respectively. Light scattering by algal cells reduces the photocurrent generated by the photodiode; the higher the $\mathrm{OD}$, the greater the reduction in photocurrent. Photodiode measurements were calibrated using the corresponding spectrophotometric OD measurements, obtained using the technique developed by Arnon. ${ }^{21}$ The $\mathrm{pO}_{2}$ electrode was calibrated to $0 \%$ oxygen in nitrogen-saturated 
water, $21 \%$ in air-saturated water and $100 \%$ in oxygen-saturated water. $\mathrm{H}_{2}$ production rates and yields were measured in situ by Membrane-Inlet Mass Spectrometry (MIMS). All measurements were recorded using a National Instruments datalogging and control system based around the National Instrument Compact RIO processor and running LabView software.

\subsection{Sulphur-deprivation techniques}

Biophotolytic $\mathrm{H}_{2}$ production only takes place under anaerobic conditions, which are initiated by sulphur-deprivation. This study makes use of the centrifugation and nutrient control techniques of sulphur-deprivation. Centrifugation was the original sulphurdeprivation process. $^{22}$ Under the centrifugation process, a C. reinhardtii culture was first grown in TAP medium until the required OD was obtained; an OD of approximately $20 \mathrm{mg} \mathrm{l}^{-1}$ of chlorophyll (Chl) is sufficient for $\mathrm{H}_{2}$ production. ${ }^{16}$ The culture was then centrifuged at $14000 \mathrm{rpm}$ for 15 minutes and a dark green pellet of algal cells formed at the bottom of the centrifugation vessel. The TAP medium was discarded and the algae were washed with, and re-suspended in, TAP-S medium in the flatplate PBR, where they proceeded to consume all available oxygen before commencing anaerobic $\mathrm{H}_{2}$ production. At least $20 \%$ of algal cells were lost during centrifugation, with additional cells damaged by the compressive stress endured during the process. ${ }^{11}$ The centrifugation process is very time, energy and labour intensive, and it is therefore impractical for any large scale application, thus motivating the need for a nutrient controlled method of $\mathrm{H}_{2}$ production. The nutrient control method of $\mathrm{H}_{2}$ production was developed in order to optimise algal growth and maximise $\mathrm{H}_{2}$ production without the need to exchange media by centrifugation at any stage of the process. This was achieved by accurately measuring algal growth rates, and acetate and sulphate uptake rates, under different growth conditions. The intention was that, given a set of initial conditions, it would be possible to predict exactly how quickly the algae would grow and precisely how much acetate and sulphate they would require. On this basis, the $\operatorname{TAP}_{12}$ growth medium was designed to ensure that all sulphur would run out just as the algae attained their maximum OD, leading to spontaneous and effective $\mathrm{H}_{2}$ production. ${ }^{19}$

\subsection{Sulphur re-insertion techniques}

$\mathrm{H}_{2}$ production duration is one of the main limitations of green algal biophotolysis and it needs to be extended significantly in order for large-scale applications to become viable. ${ }^{23}$ Since the availability of sulphur governs the rate of photosynthesis, and consequently the $\mathrm{pO}_{2}$ levels of the $C$. reinhardtii culture, sulphur re-addition following the onset of anaerobic conditions should encourage algal cell recovery, and enable additional $\mathrm{H}_{2}$ production over a longer time frame. Two different sulphur re-addition techniques were used in this study: sulphur dilution and sulphur feed. The objective of the sulphur dilution method was to initiate multiple cycles of algal growth and $\mathrm{H}_{2}$ production. The $C$. reinhardtii culture was initially allowed to complete one full cycle of growth and $\mathrm{H}_{2}$ production. This culture was subsequently diluted with a sulphur-optimised medium in an attempt to induce a secondary cycle of growth and $\mathrm{H}_{2}$ production.
The objective of the sulphur feed technique was long-term operation of the flat-plate PBR in chemostat mode. A micromolar sulphur feed was applied to $C$. reinhardtii cultures while they were still producing $\mathrm{H}_{2}$. This sulphur feed needed to contain sufficient nutrients for $C$. reinhardtii culture growth without raising photosynthesis rates too high and stopping anaerobic $\mathrm{H}_{2}$ production.

\subsection{Analysis techniques}

Ion chromatography (IC) was used to measure sulphate uptake during $C$. reinhardtii growth. IC was carried out using the 882 Compact IC Plus instrument with an 863 Compact Autosampler (Metrohm) running the MagIC Net software. The technique used a Metrosep A Supp 10 separation column, which is capable of analysing compounds whose concentrations differ widely. The concentrations of compounds leaving the column were measured by means of a conductivity detector. The sodium-based anion solution (liquid phase) consisted of $3.2 \mathrm{mM}$ sodium carbonate, $1.0 \mathrm{mM}$ sodium bicarbonate and $5.0 \mu \mathrm{M}$ sodium perchlorate. Deionised water was used as the rinse and 0.1 M sulphuric acid as the regenerator. The liquid phase was pumped through the instrument using a peristaltic pump at a flow rate of $1.0 \mathrm{ml} \mathrm{min}{ }^{-1}$. Algal samples were spun down in a mini-centrifuge at $13000 \mathrm{rpm}$ for $10 \mathrm{~min}$ : the purified medium was used for IC and High-Performance Liquid Chromatography (HPLC) and the algal pellet was discarded. Samples of $25 \mu \mathrm{l}$ were injected into the IC instrument. The anion concentrations corresponded to the area occupied by their respective peaks. These areas were calibrated against concentration using CertiPUR Anion Multi-Element Standards (Merck) at concentrations of $1 \mathrm{ppm}$, $10 \mathrm{ppm}, 50 \mathrm{ppm}, 100 \mathrm{ppm}$ and $200 \mathrm{ppm}$. The retention time for the sulphate anion was $22.0 \mathrm{~min}$. HPLC was used to measure acetate uptake during algal growth. It was performed using the Hewlett Packard Series 1050 instrument running HPCORE ChemStation software. The technique was based on a wellestablished procedure designed to investigate the concentration of fermentative products such as organic acids and alcohols. ${ }^{24}$ The Aminex HPX-87H Ion Exchange Column from BioRad (HPLC Organic Acid Analysis Column) was used at a temperature of 58.0 ${ }^{\circ} \mathrm{C}$ together with a BioRad guard column. Compounds were detected by means of an ultra-violet detector operating at $210 \mathrm{~nm}$. The liquid phase consisted of $4 \mathrm{mM}$ sulphuric acid, which was peristaltically pumped through the column at a flow rate of $0.6 \mathrm{ml} \mathrm{min}{ }^{-1}$. Samples of $50 \mu \mathrm{l}$ were injected into the instrument by means of an autosampler. A strong acetate peak was observed with a residence time of $15.1 \mathrm{~min}$ and the peak area was calibrated against acetate concentration using $20000 \mathrm{ppm}, 5000 \mathrm{ppm} 1000 \mathrm{ppm}$ and $200 \mathrm{ppm}$ standards prepared from $99.99 \%$ pure acetic acid.

\section{Results and discussion}

\subsection{Flat-plate photobioreactor design}

In order to improve the efficiency and duration of biohydrogen production, it was first necessary to design a PBR that would effectively facilitate green algal growth and $\mathrm{H}_{2}$ production, 
while also enabling accurate measurement of $\mathrm{H}_{2}$ production rates and yields. A vertical flat-plate laboratory-scale PBR has already been designed, constructed, commissioned and operated (Fig. 1). ${ }^{19}$ This flat-plate reactor geometry was chosen due to its superior surface-to-volume ratio, which results in the highest observed photochemical efficiencies for $\mathrm{H}_{2}$ production. ${ }^{25}$ The PBR body consists of two separate rectangular compartments (Fig. 1a). The primary compartment holds the algal culture and contains the key measuring instruments, including the MIMS system developed for in situ $\mathrm{H}_{2}$ measurement and extraction. MIMS extracts $\mathrm{H}_{2}$ from the algal culture by the process of pervaporation. ${ }^{26} \mathrm{H}_{2}$ molecules are absorbed onto the surface of a polydimethylsiloxane (PDMS) membrane, they permeate through the membrane material and are desorbed into the argon carrier gas on the other side, which transports them to a mass spectrometer for analysis. This study used the compact quadrupole Pfeiffer Vacuum Prisma mass spectrometer, running Quadstar 422 software. The mass spectrometer ion current signal was calibrated against known $\mathrm{H}_{2}$ concentrations in the primary compartment. The maximum $\mathrm{H}_{2}$ pervaporation rate through the PDMS membrane has been calculated to be $9.3 \mathrm{ml} \mathrm{h}^{-1} .^{20}$ Since this is significantly higher than the $2.0 \mathrm{ml} \mathrm{h}^{-1}$ (per litre of culture) C. reinhardtii $\operatorname{cc} 124 \mathrm{H}_{2}$ production rate, ${ }^{27}$ any $\mathrm{H}_{2}$ produced by the algae is immediately extracted by MIMS. The mass spectrometer measurement therefore directly corresponds to the $\mathrm{H}_{2}$ production rate within the flat-plate PBR. Turbulent culture mixing within the primary compartment is achieved by a circulating gas-lift system, emanating from a dual-entry sparger located at the bottom of the compartment, powered by a gas diaphragm pump (Fig. 1b). While being circled between the gas diaphragm pump and the PBR primary compartment, the resident gas passes through a water displacement and pressure release system. A coolwhite light-emitting diode (LED) array provides uniform, coherent, non-heating illumination to the system. The secondary compartment is filled with water or aqueous solution. The liquid is heated to control the temperature in the primary compartment; coloured ions can also be dissolved in the water to modulate the wavelength incident on the primary compartment.

\section{2 $\quad \mathrm{H}_{2}$ Production by nutrient control}

$\mathrm{H}_{2}$ production by $C$. reinhardtii takes place under anaerobic conditions, which may be imposed metabolically by depriving the algae of sulphur. Sulphur-deprivation normally requires the spatial and temporal separation of the algal growth and $\mathrm{H}_{2}$ production stages. This would typically require separate PBRs for each stage as well as a costly and energy-intensive medium exchange technique such as centrifugation, making the process difficult to scale up. In a previous study, the nutrient control method of hydrogen production has been developed to overcome these limitations. ${ }^{19}$ In order to develop the nutrient control method, sulphate and acetate uptake rates during algal growth were investigated under different illumination conditions. The experiment was repeated in various PBR geometries in order to determine a reactor-independent relationship between the algal growth and nutrient consumption kinetics. Higher light intensities (or higher algal growth rates) imply higher photosynthesis rates, increasing the turnover of the PSII repair mechanism and therefore increasing the demand for sulphur, both in terms of the sulphate uptake rate and of the total quantity of sulphur required. Similarly, higher light intensities (higher algal growth rates) lead to an increase in the acetate uptake rate. On the other hand, they also result in increased carbon dioxide fixation by the Calvin cycle and a corresponding decrease in the total quantity of acetate consumed. ${ }^{19}$ Using the relationships between the algal growth rate and nutrient uptake rates, the initial sulphur and acetate concentrations of the algal medium were optimised so that these nutrients run out at the exact moment when the maximum algal OD is reached. In this study, a starting sulphate concentration of $28.0 \mathrm{mg} \mathrm{l}^{-1}$ and a starting acetate concentration of $2550 \mathrm{mg} \mathrm{l}^{-1}$ were used to achieve algal growth up to an OD of as much as $35.9 \mathrm{mg} \mathrm{l}^{-1} \mathrm{Chl}$ under nutrient-optimised conditions (Fig. 2). All acetate was used up at a specific uptake rate of $0.103 \mathrm{~h}^{-1}$ (Fig. 2a) and all sulphate was used up at a specific uptake rate of $0.110 \mathrm{~h}^{-1}$ (Fig. 2b) over a period of 104 hours. Following the complete consumption of sulphate, anaerobic conditions were established (Fig. 2c). The subsequent $\mathrm{H}_{2}$ production stage lasted for approximately 170 hours, with a maximum $\mathrm{H}_{2}$ production rate of $1.52 \mathrm{ml} \mathrm{H}_{2} \mathrm{l}^{-1} \mathrm{~h}^{-1}$ and a $\mathrm{H}_{2}$ yield of $119.8 \mathrm{ml} \mathrm{H}_{2} \mathrm{l}^{-1}$. During this time, the OD eventually reduced to $23.4 \mathrm{mg} \mathrm{l}^{-1} \mathrm{Chl}$ as a result of catabolic processes (Fig. 2d). The maximum $\mathrm{H}_{2}$ production rate by nutrient control $\left(1.52 \mathrm{ml} \mathrm{H}_{2} \mathrm{l}^{-1} \mathrm{~h}^{-1}\right)$ was higher than the maximum $\mathrm{H}_{2}$ production rate by centrifugation (1.11 $\mathrm{ml} \mathrm{H}_{2} \mathrm{l}^{-1} \mathrm{~h}^{-1}$ ) under the same experimental conditions. ${ }^{19}$ The reason for this is that the algal culture in the nutrientcontrolled experiment had a higher OD at the moment of sulphur deprivation, since cells were not lost or damaged by a nutrient exchange procedure. ${ }^{16}$ There were therefore more cells, and hence more PSII reaction centres, available for $\mathrm{H}_{2}$ production. The nutrient control method of sulphur-deprivation was easy to implement and it proved superior to its counterparts based on all the relevant $\mathrm{H}_{2}$ production criteria, indicating that it could be the technique of choice for $\mathrm{H}_{2}$ production experiments as well as for scaled-up applications of biophotolytic $\mathrm{H}_{2}$ production. It creates the opportunity for automated and spontaneous $\mathrm{H}_{2}$ production using a single PBR.

\subsection{Extending $\mathrm{H}_{2}$ production duration by sulphur dilution}

The nutrient control method does not address the main shortcoming of green algal $\mathrm{H}_{2}$ production, namely the short duration of the $\mathrm{H}_{2}$ production process. However, it was possible to use the nutrient-optimised $\mathrm{TAP}_{12}$ medium to facilitate sulphur re-insertion by dilution in order to drive long-term biohydrogen production. The aim of the sulphur dilution experiments was to follow up a complete $\mathrm{H}_{2}$ production cycle with subsequent C. reinhardtii cell recovery and secondary $\mathrm{H}_{2}$ production cycles. The initial $\mathrm{H}_{2}$ production period was initiated by centrifugation. Upon the completion of a full $\mathrm{H}_{2}$ production cycle, sulphur (and acetate) were re-inserted by means of a dilution with $\mathrm{TAP}_{12}$ medium. A dilution of $95 \%$ was used, which implies that $5 \% \mathrm{v} / \mathrm{v}$ of the depleted $C$. reinhardtii culture was diluted with $95 \% \mathrm{v} / \mathrm{v}$ of fresh $\mathrm{TAP}_{12}$ medium. Since the $\mathrm{TAP}_{12}$ medium 
(a) LED array

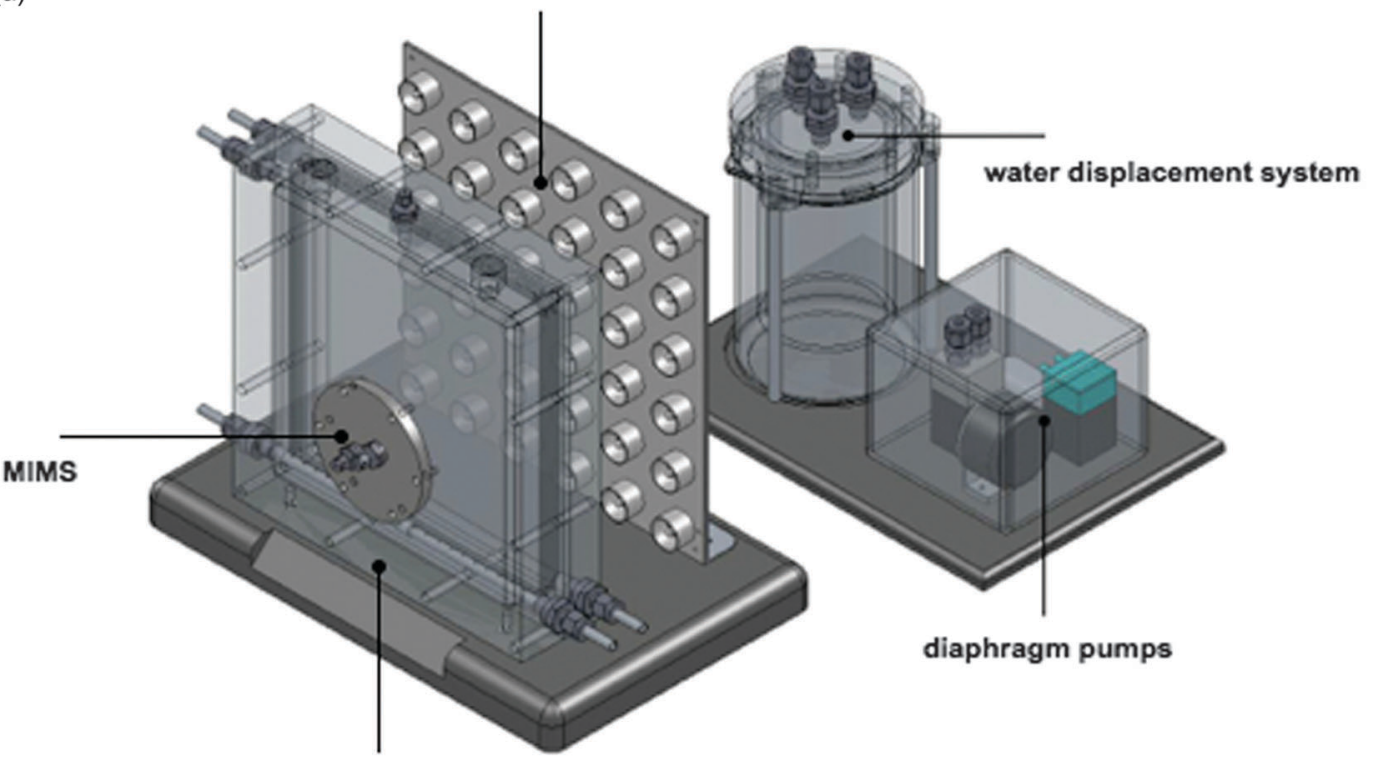

flat-plate PBR body

(b)

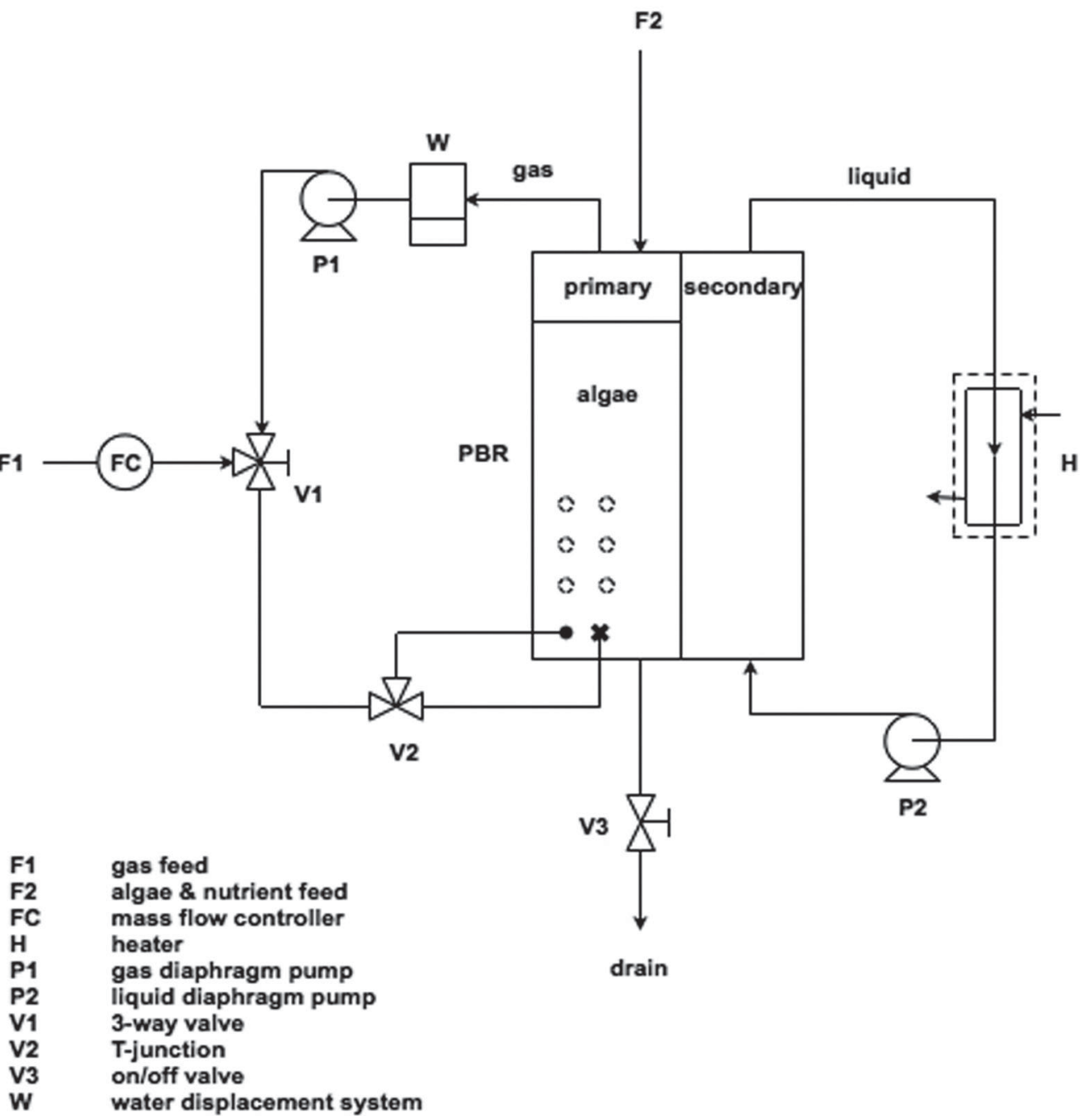

Fig. 1 Flat-plate PBR designed to facilitate biophotolytic $\mathrm{H}_{2}$ production: (a) photobioreactor appearance and assembly in solid works; (b) technical diagram of the PBR mixing and circulation systems. 


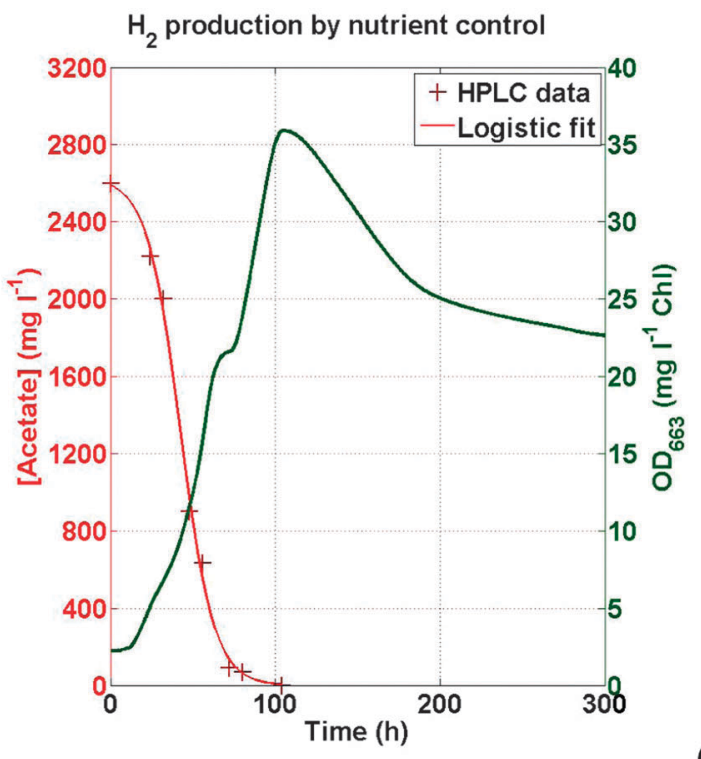

(a)

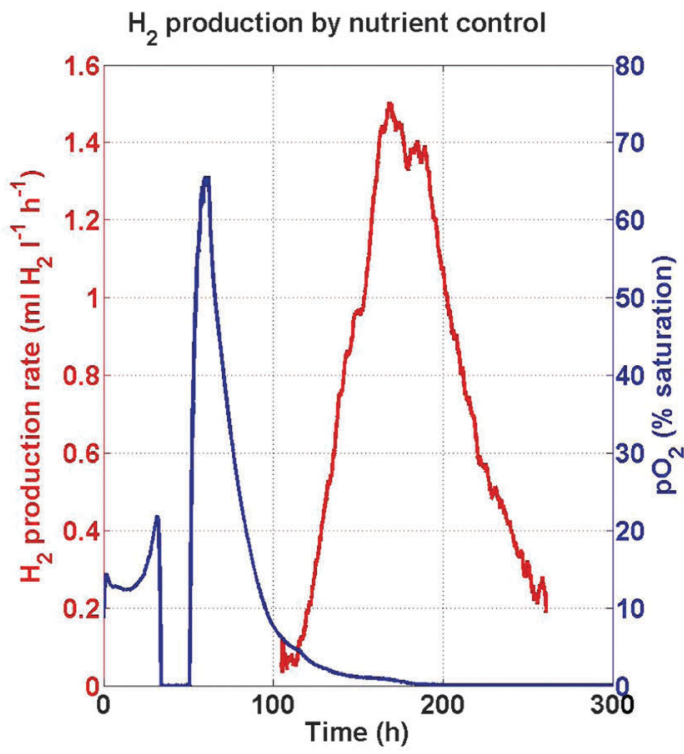

(c)

(d) (b)
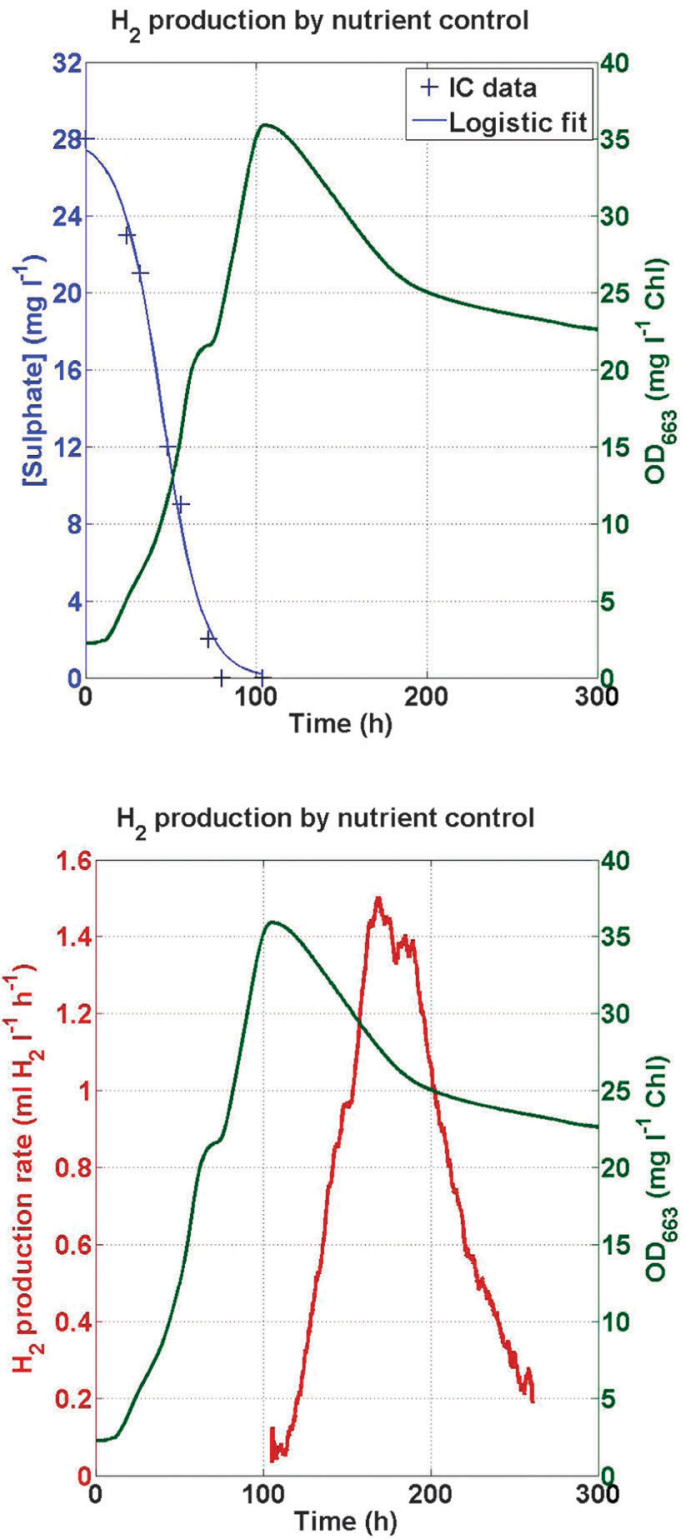

Fig. 2 C. reinhardtii $\mathrm{cc} 124 \mathrm{H}_{2}$ production by nutrient control in the flat-plate PBR: (a) acetate uptake during algal growth; (b) sulphate uptake during algal growth; (c) oxygen consumption followed by $\mathrm{H}_{2}$ production; (d) algal growth followed by $\mathrm{H}_{2}$ production and biomass catabolism.

was developed to provide optimum batch sulphate and acetate feed for an algal culture growing at $12 \mathrm{~W} \mathrm{~m}^{-2}$ in the flat-plate $\mathrm{PBR}$, it follows that a $95 \% \mathrm{v} / \mathrm{v}$ dilution with $\mathrm{TAP}_{12}$ should provide the optimal nutrient feed to encourage the recovery of $95 \%$ of the C. reinhardtii culture growing under the same ambient conditions. The 95\% dilution experiment is shown in Fig. 3. The $\mathrm{pO}_{2}$ dropped to zero within 18 hours following sulphur-deprivation, resulting in anaerobic $\mathrm{H}_{2}$ production with a maximum $\mathrm{H}_{2}$ production rate of $1.03 \mathrm{ml} \mathrm{H}_{2} \mathrm{l}^{-1} \mathrm{~h}^{-1}$ (Fig. 3a). This $\mathrm{H}_{2}$ production stage lasted over a period of 132 hours; the OD reduced from $28.1 \mathrm{mg} \mathrm{l}^{-1} \mathrm{Chl}$ to $21.5 \mathrm{mg} \mathrm{l}^{-1} \mathrm{Chl}$ during this time (Fig. 3b). Following 150 hours of experiment, the depleted C. reinhardtii culture was diluted with $95 \% \mathrm{TAP}_{12}$. This dilution reduced the OD from $21.5 \mathrm{mg} \mathrm{l}^{-1} \mathrm{Chl}$ to $6.1 \mathrm{mg} \mathrm{l}^{-1} \mathrm{Chl}$, but it supplied the cells with $29.0 \mathrm{mg} \mathrm{l}^{-1}$ of sulphate (Fig. 3c) and $3130 \mathrm{mg} \mathrm{l}^{-1}$ of acetate (Fig. 3d). During the cell recovery stage, which lasted for 80 hours, the entire nutrient supply was consumed and the algal OD eventually increased to $31.2 \mathrm{mg} \mathrm{l}^{-1} \mathrm{Chl}$, higher than the original OD following centrifugation. The $\mathrm{pO}_{2}$ remained at zero throughout the recovery period. Following the consumption of sulphur, the algae immediately entered a secondary $\mathrm{H}_{2}$ production period with a maximum $\mathrm{H}_{2}$ production rate of $1.13 \mathrm{ml} \mathrm{H}_{2} \mathrm{l}^{-1} \mathrm{~h}^{-1}$ and a duration of 145 hours. The OD decreased from $31.2 \mathrm{mg} \mathrm{l}^{-1} \mathrm{Chl}$ to $23.1 \mathrm{mg} \mathrm{l}^{-1} \mathrm{Chl}$ in this time. The $95 \%$ dilution proved to be an effective way of inducing a secondary $\mathrm{H}_{2}$ production period. The advantage of a $95 \%$ dilution over starting a new experiment is that it allows prolonged cyclic $\mathrm{H}_{2}$ production within a single PBR without the need to 


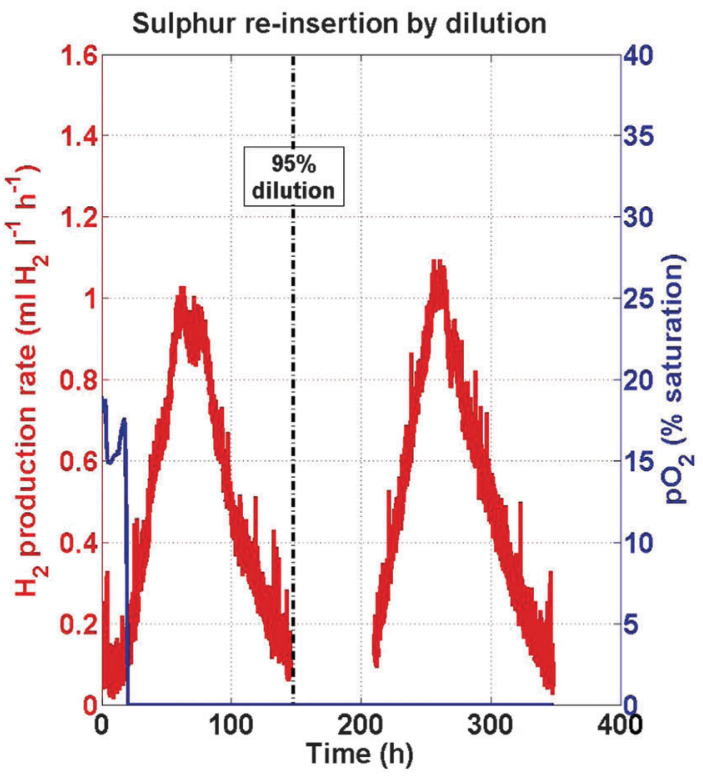

(a)

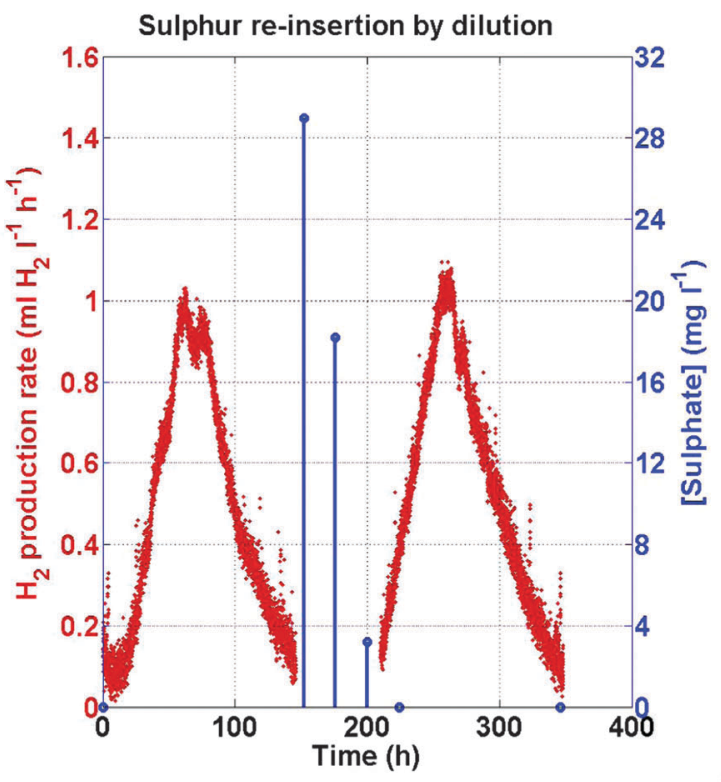

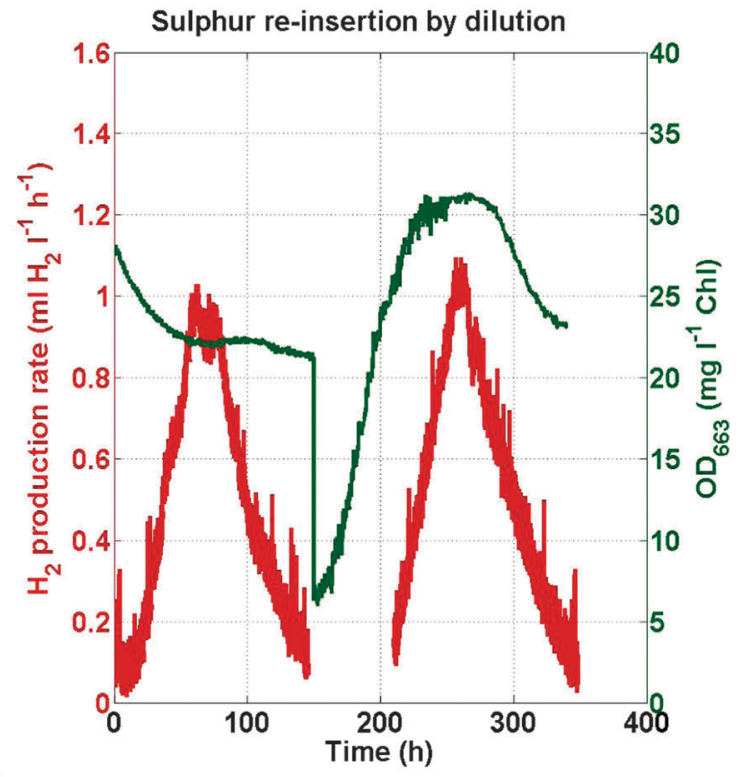

(b)

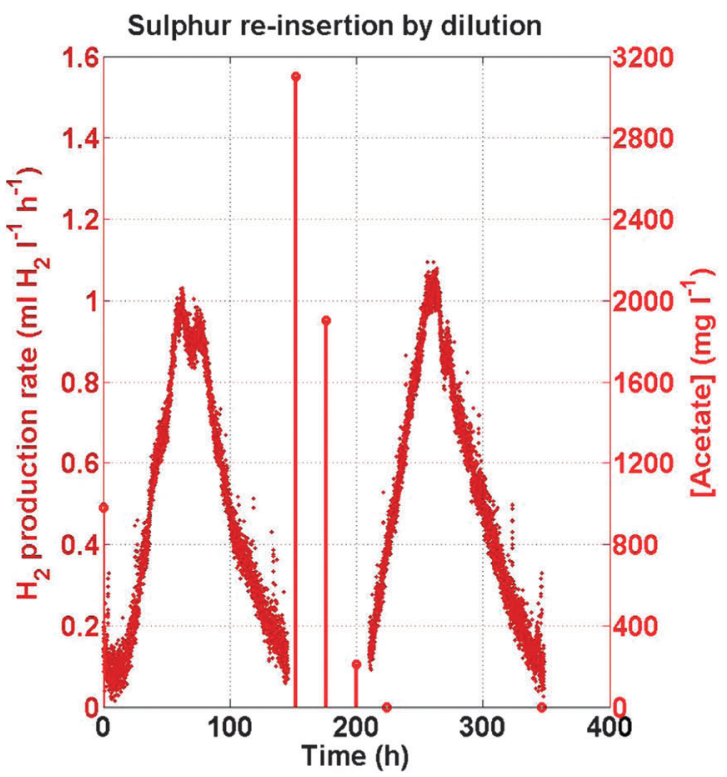

Fig. 3 Cyclic $\mathrm{H}_{2}$ production by C. reinhardtii cc124 in the flat-plate PBR following sulphur re-insertion by $95 \%$ dilution: (a) $\mathrm{H}_{2}$ production and $\mathrm{pO}_{2}$ against time; (b) $\mathrm{H}_{2}$ production and algal $\mathrm{OD}$ against time; (c) $\mathrm{H}_{2}$ production and sulphate concentration against time; (d) $\mathrm{H}_{2}$ production and acetate concentration against time.

completely empty and clean it. The lack of a $\mathrm{pO}_{2}$ recovery also decreases the amount of time required to initiate the secondary $\mathrm{H}_{2}$ production stage because it eliminates a time-consuming oxygen consumption stage. The secondary $\mathrm{H}_{2}$ production stage was superior to the primary $\mathrm{H}_{2}$ production stage because there was a higher initial algal cell density, since the secondary $\mathrm{H}_{2}$ production stage was initiated by nutrient control rather than centrifugation.

\subsection{Extending $\mathrm{H}_{2}$ production duration by sulphur feed}

The aim of the sulphur feed technique was to operate the flatplate PBR in chemostat mode for sulphate in order to achieve prolonged $\mathrm{H}_{2}$ production. A dosing pump was used to inject micromolar sulphur feed into the sealed flat-plate PBR during the anaerobic $\mathrm{H}_{2}$ production stage. The sulphur feed was applied towards the beginning of the $\mathrm{H}_{2}$ production stage, at a point when the $\mathrm{H}_{2}$ production rate was still increasing. This timing was chosen in order to maximise the observability of any negative effect of the sulphur feed on the algal $\mathrm{H}_{2}$ production rate by MIMS. The intention was to keep sulphate concentrations of the algal medium below $2 \mathrm{ppm}$, the minimum tolerance level for $\mathrm{H}_{2}$ production. ${ }^{28}$ At the same time, it was necessary to provide a small acetate feed so that the culture would not fail due to a lack of carbon. Since the resulting nutrient feed was highly acidic, the HEPES (4-(2-hydroxyethyl)-1-piperazineethanesulfonic acid) buffer was used. The sulphate consumption rate 


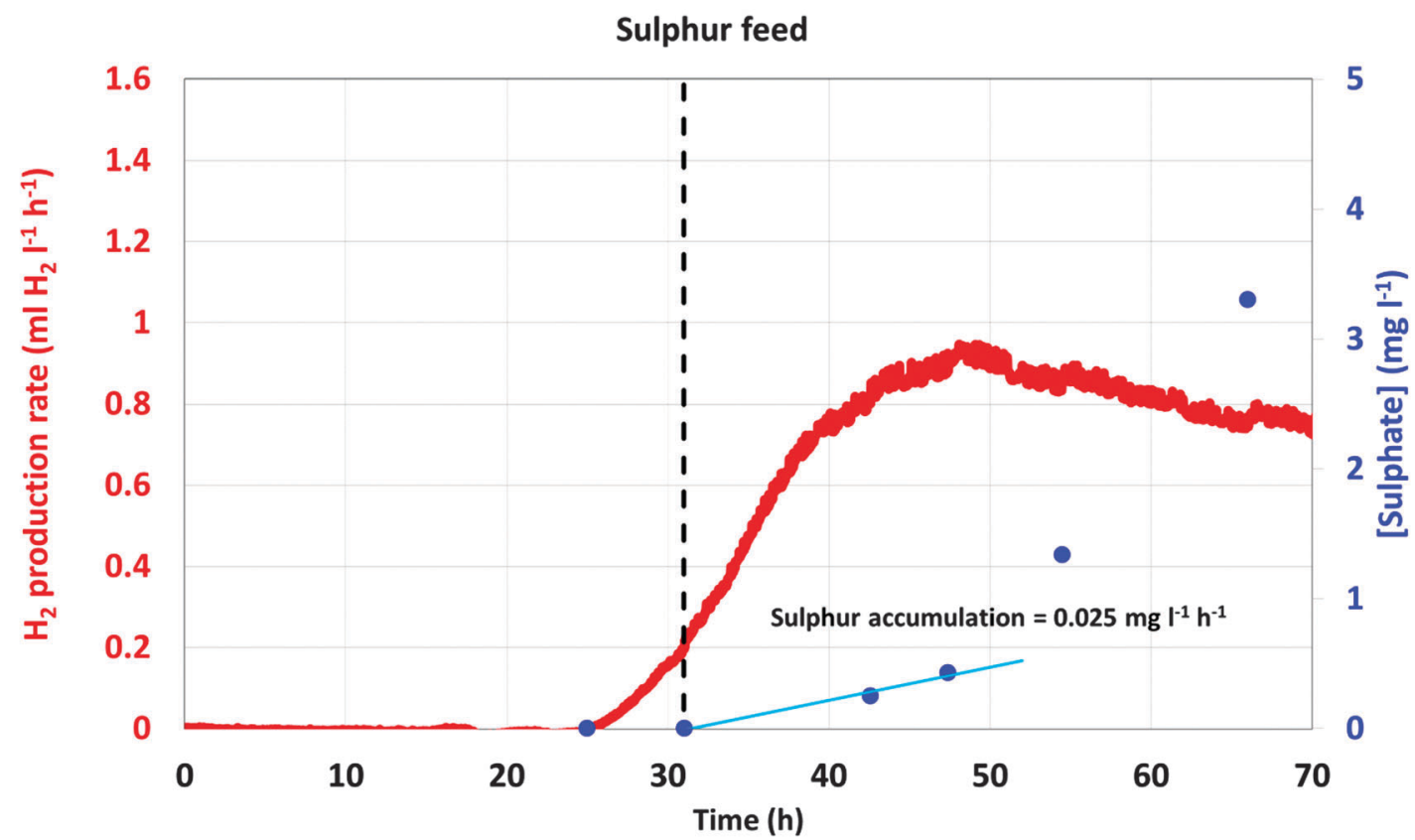

Fig. 4 Sulphur accumulation rate $\left(0.025 \mathrm{mg} \mathrm{SO}_{4}^{-2} \mathrm{I}^{-1} \mathrm{~h}^{-1}\right)$ in the algal growth medium following the activation of a $0.067 \mathrm{mg} \mathrm{SO}_{4}^{-2} \mathrm{I}^{-1} \mathrm{~h}^{-1}$ sulphur feed during anaerobic $\mathrm{H}_{2}$ production by $\mathrm{C}$. reinhardtii $\mathrm{cc} 124$ in the flat-plate PBR. The sulphur uptake rate by algal cells was $0.042 \mathrm{mg} \mathrm{SO}_{4}^{-2} \mathrm{I}^{-1} \mathrm{~h}^{-1}$, which is therefore the optimum sulphur feed rate.

by C. reinhardtii cc124 was calculated by subtracting the rate of sulphate accumulation in the algal growth medium from the corresponding sulphate delivery rate. An initial sulphur feed experiment was carried out using an arbitrary sulphate delivery rate of $0.067 \mathrm{mg} \mathrm{SO}_{4}{ }^{-2} \mathrm{l}^{-1} \mathrm{~h}^{-1}$ (Fig. 4). In the first 20 hours $(t=$ $30 \mathrm{~h}$ to $t=50 \mathrm{~h}$ ) following activation of the dosing pump (vertical dotted line), the sulphate concentration increased linearly, but it remained low enough not to inhibit $\mathrm{H}_{2}$ production. The rate of sulphate accumulation during this period was calculated to be $0.025 \mathrm{mg} \mathrm{SO}_{4}{ }^{-2} \mathrm{l}^{-1} \mathrm{~h}^{-1}$, giving a C. reinhardtii sulphate consumption rate of $0.042 \mathrm{mg} \mathrm{SO}{ }_{4}^{-2} \mathrm{l}^{-1} \mathrm{~h}^{-1}$. This value was used in subsequent experiments to set the sulphate delivery rate to exactly meet cellular sulphur requirement, without disrupting $\mathrm{H}_{2}$ production activities. In the case of Fig. 4, the growth medium becomes overloaded with sulphate $(t=50 \mathrm{~h})$, the $\mathrm{H}_{2}$ production rate collapses, and the sulphate accumulation rate in the growth medium begins to match the sulphate delivery rate. Fig. 5 illustrates an experiment carried out using the optimised sulphur feed rate of $0.042 \mathrm{mg} \mathrm{SO}_{4}{ }^{-2} \mathrm{l}^{-1} \mathrm{~h}^{-1}$. A fully-grown C. reinhardtii cc124 culture was centrifuged and re-suspended in TAP-S medium and later cultivated in the flatplate PBR at $12 \mathrm{~W} \mathrm{~m}^{-2}$ and $25{ }^{\circ} \mathrm{C}$. Anaerobic conditions were established by the sulphur-deprived culture approximately 24 hours into the experiment, resulting in the onset of $\mathrm{H}_{2}$ production. The dosing pump was activated shortly after $(t=29 \mathrm{~h})$. The results show that the PBR operated as a chemostat for both sulphate (Fig. 5a) and acetate (Fig. 5b) until the end of the experiment. The $C$. reinhardtii culture remained anaerobic (Fig. 5c) and continued to produce $\mathrm{H}_{2}$ over a period of 130 hours (more than 5 days) despite the presence of a continuous lowconcentration sulphur feed. Nevertheless, the $\mathrm{H}_{2}$ production rate steadily decreased following approximately 120 hours of experiment. The decrease in $\mathrm{H}_{2}$ production follows on from a continuous decline in the algal cell density under anaerobic conditions (Fig. 5d). Sulphur deprivation is therefore not the sole reason for the termination of $\mathrm{H}_{2}$ production in $C$. reinhardtii following 5-7 days of experiment. The prolonged exposure to an anaerobic environment likely results in irreversible changes in the intracellular metabolism of $C$. reinhardtii. ${ }^{7}$ The algal culture requires a secondary growth phase, such as the one shown in Fig. 3b, to fully recover prior to commencing further $\mathrm{H}_{2}$ production. The sulphur dilution technique is therefore the only scalable option for extended $\mathrm{H}_{2}$ production at this time.

\subsection{Photobioreactor concept for $\mathrm{H}_{2}$ production scale-up}

The nutrient control technique of $\mathrm{H}_{2}$ production and the dilution technique of sulphur re-insertion were developed with the objective of enabling more effective and scalable biophotolytic $\mathrm{H}_{2}$ production. The next step is to design a scaled-up PBR in order to put these techniques into practice and investigate long-term spontaneous $\mathrm{H}_{2}$ production by C. reinhardtii cultures under realworld conditions. This could be achieved by pursuing the concept of cyclic $C$. reinhardtii growth and $\mathrm{H}_{2}$ production in an outdoor semi-continuous PBR (Fig. 6). This conceptual design is a suggestion of what a near-continuous large scale process might look like based on the findings of the small scale research conducted in this study and in previous publications. ${ }^{19,20}$ The application consists of several PBRs, each facilitating cyclic $\mathrm{H}_{2}$ production and algal recovery by sulphur dilution, with a time lag between the PBRs, so that the algal culture in each reactor is at a different stage of the cyclic sulphur dilution process. Fig. 6 illustrates this concept using three PBRs. In the first reactor (R1), fresh nutrients 


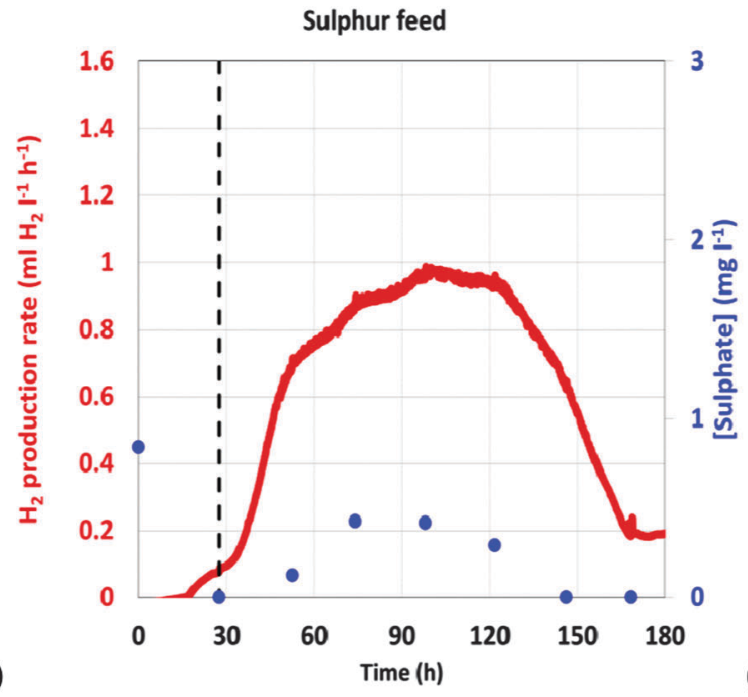

(a)

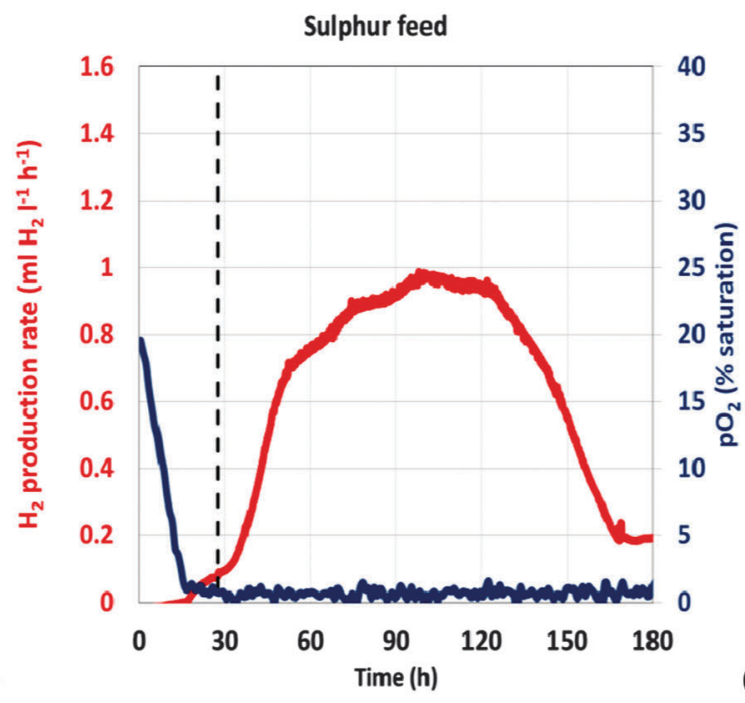

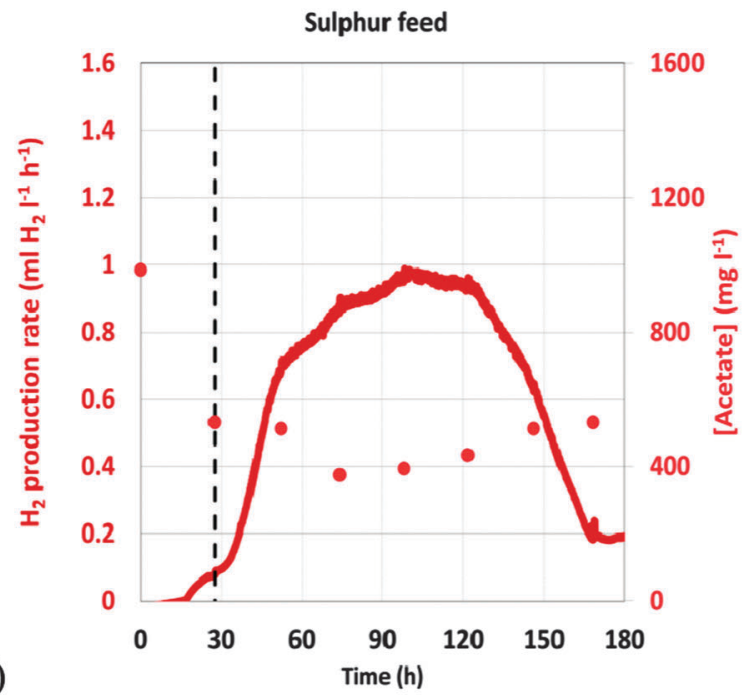

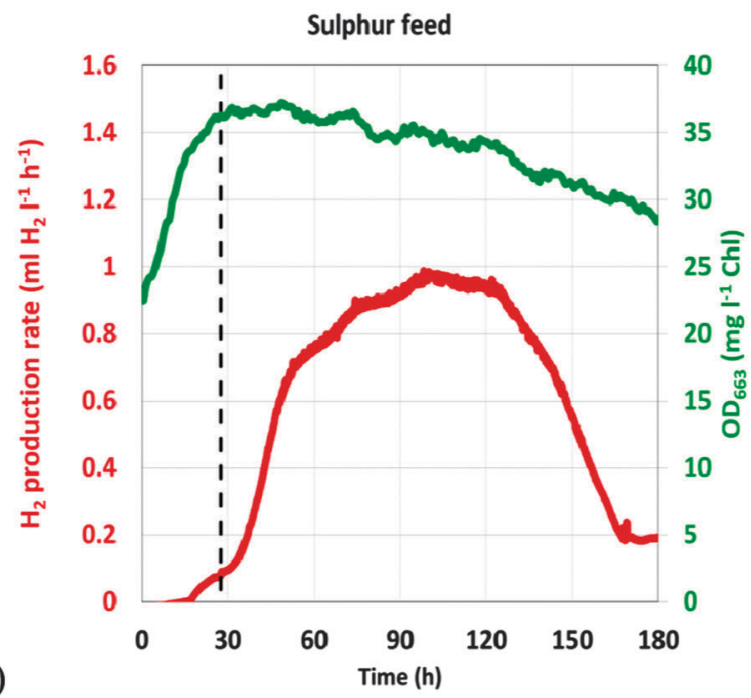

Fig. $5 \mathrm{H}_{2}$ production by C. reinhardtii $\mathrm{cc} 124$ in the flat-plate PBR under a sulphur feed rate of $0.042 \mathrm{mg} \mathrm{SO}_{4}{ }^{-2} \mathrm{I}^{-1} \mathrm{~h}^{-1}$ : (a) PBR operates as a chemostat for sulphate and the sulphate concentration remains below the $1 \mathrm{mg} \mathrm{SO}_{4}{ }^{-2} \mathrm{I}^{-1}$ limit, enabling anaerobic $\mathrm{H}_{2}$ production; (b) PBR operates as a chemostat for acetate; (c) anaerobic conditions $\left(\mathrm{pO}_{2}=0\right)$ are maintained throughout the experiment; (d) optical density decreases despite the continuous sulphate and acetate feed, eventually resulting in the termination of $\mathrm{H}_{2}$ production.

have been delivered to a depleted $C$. reinhardtii culture via valve $\mathrm{V} 1$ and the algal recovery process is about to begin. In R2, the algal culture has grown to its final OD, the nutrients have been consumed and $\mathrm{H}_{2}$ production is about to commence. Finally, in $\mathrm{R} 3, \mathrm{H}_{2}$ production has been completed and algal biomass is about to be harvested via valve V6. Given a sufficiently large number of reactors, it is possible to achieve continuous biomass and $\mathrm{H}_{2}$ production using this system. A gravity-assisted flow system is used for biomass harvesting (green arrows) and nutrient delivery (blue arrows). Algal biomass flows down into a cross-flow micro-filter, ${ }^{29}$ which separates the usable solid mass from the recyclable liquid growth medium. A nutrient concentration detector measures the nutrient composition of the recycled growth medium, which is enhanced using fresh nutrients delivered by a dosing pump. The nutrient feed is raised above the PBR level by means of an energy-efficient Archimedes screw; ${ }^{30}$ the nutrient feed then flows down into the appropriate PBRs. The gas flow system has been labelled with red arrows. Pre-processed flue gas, the waste gas output of a fossil fuel power station, delivers concentrated carbon dioxide and mixing to all the PBRs, while also picking up any $\mathrm{H}_{2}$ that has been produced. The $\mathrm{H}_{2}$ product is separated using a $\mathrm{H}_{2}$-permeable membrane, similar to that used in the MIMS system, and the majority of the carbon dioxide is sequestered by the growing algal culture. The system described in Fig. 6 therefore provides $\mathrm{H}_{2}$ production and extraction, solid biomass production and extraction, nutrient control and recycling, and temporary carbon dioxide sequestration.

The individual reactor units of this continuous batch PBR could be manufactured economically from high-density polymers in a process similar to the manufacture of commercial plastic bags. The choice of PBR polymer is a compromise 


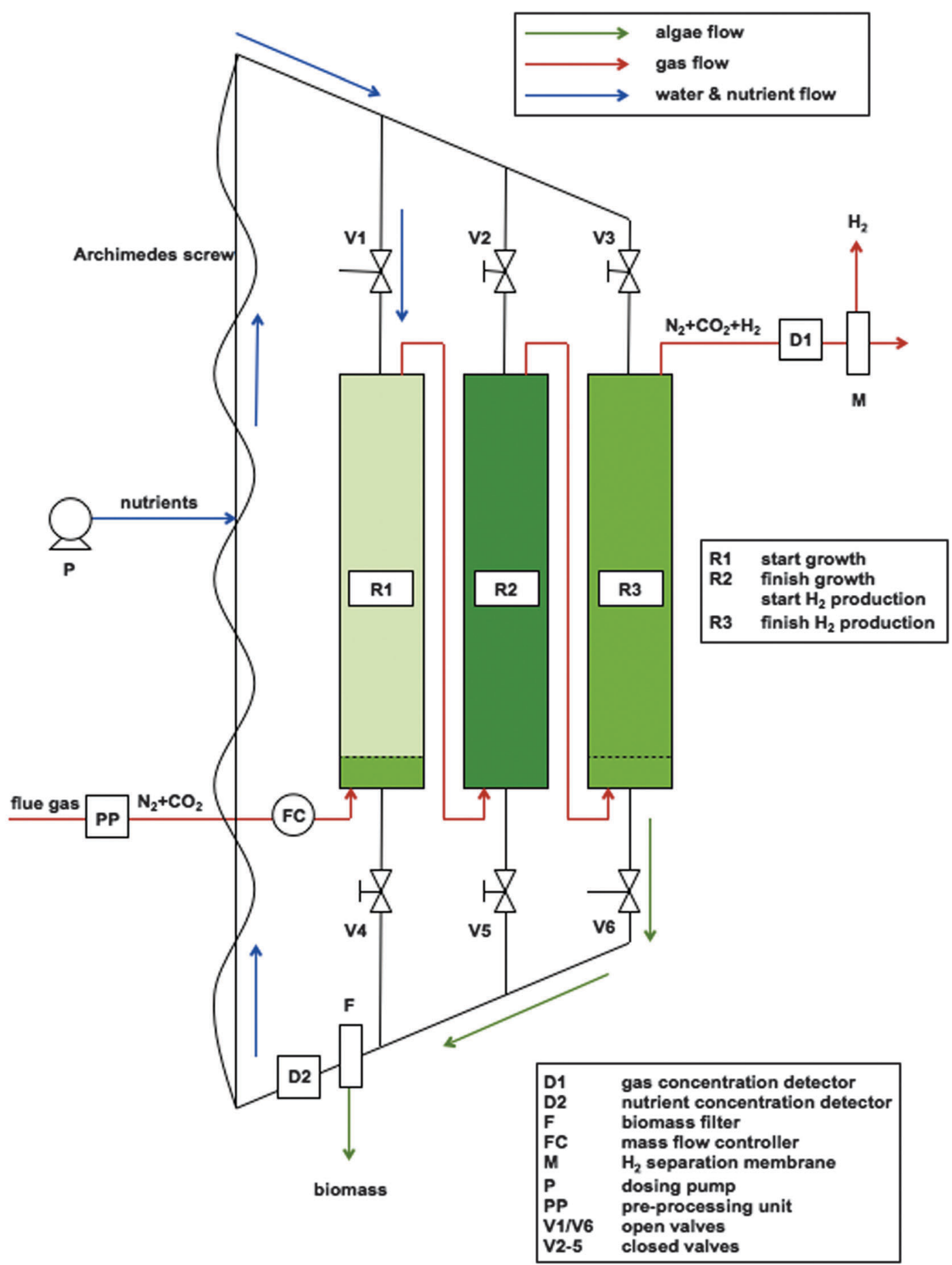

Fig. 6 Proposed PBR concept that would enable biophotolytic $\mathrm{H}_{2}$ production scale-up.

between its ability to retain $\mathrm{H}_{2}\left(\mathrm{H}_{2}\right.$ permeability) and the corresponding cost and available production capacity. Another important factor is the ability to process the polymer into a blow film by plastic extrusion. Final consideration needs to be given to weathering, photo-degradation and yellowing of the material, which results in reduced irradiation of the algal culture. This remains a poorly understood are with many interacting parameters, despite much commercial and academic work regarding natural and accelerated testing methodologies. ${ }^{31}$ The $\mathrm{H}_{2}$ permeability of selected polymers of interest for PBR construction is given in Table $1 .^{32,33}$ PVDF has the lowest $\mathrm{H}_{2}$ permeability, but it is fluorinated and therefore too expensive. PET also has a low $\mathrm{H}_{2}$ permeability, it is structurally robust and large-scale manufacturing is well-established (production of food and drink containers). PET thickness is constantly being reduced, but it is not yet flexible enough to produce plastic bags. PVC and HDPE are more affordable 
Table $1 \mathrm{H}_{2}$ permeability in selected polymers of interest for PBR construction

\begin{tabular}{|c|c|c|c|}
\hline Polymer name & Abbreviation & $\begin{array}{l}\mathrm{H}_{2} \text { permeability } \\
\left(\text { Barrers }^{a}\right)\end{array}$ & Ref. \\
\hline $\begin{array}{l}\text { Polyvinylidene } \\
\text { fluoride }\end{array}$ & PVDF & 0.32 & Massey (2003) \\
\hline $\begin{array}{l}\text { Polyethylene } \\
\text { terephthalate }\end{array}$ & PET & 0.39 & Massey (2003) \\
\hline $\begin{array}{l}\text { Polyvinyl } \\
\text { chloride }\end{array}$ & PVC & 1.38 & Toi et al. (1980) \\
\hline $\begin{array}{l}\text { High density } \\
\text { polyethylene }\end{array}$ & HDPE & 2.95 & Toi et al. (1980) \\
\hline \multicolumn{4}{|c|}{$\begin{array}{l}{ }^{a} \text { 1 Barrer }=10^{-11}\left(\mathrm{~cm}^{3} \mathrm{H}_{2}\right) \mathrm{cm}^{2} \mathrm{~cm}^{-3} \mathrm{~s}^{-1} \mathrm{mmHg}^{-1}=3.348 \times \\
10^{-19} \mathrm{kmol} \mathrm{m} \mathrm{m}^{-2} \mathrm{~s}^{-1} \mathrm{~Pa}^{-1} .\end{array}$} \\
\hline
\end{tabular}

and can be blown as flexible films to produce plastic bags. A quantitative analysis of the polymer cost against the cost of $\mathrm{H}_{2}$ lost by diffusion would need to be carried out for PVC and HDPE to make a final decision regarding the choice of polymer used for PBR construction.

The plastic bag PBR units should have a working volume of the order of 100-200 litres, which represents scale-up by a factor of 100 from most laboratory PBR systems; nevertheless, it remains reasonable to expect that such PBR units could be maintained by a single vigilant operator. An important recommendation is to begin with a single plastic bag unit in order to investigate its applicability to $\mathrm{H}_{2}$ production at this scale, before expanding operations to a complete semi-continuous PBR system involving multiple plastic bag units. It is critical to investigate $C$. reinhardtii culture adaptation to the plastic bag environment as well as the challenges associated with operating the semi-continuous PBR at this stage. In terms of culture adaptation, the main consideration involves the practicality of growing $C$. reinhardtii cultures in plastic bags, under natural illumination and ambient temperatures, and using an industrial carbon dioxide feed. Potential challenges could include leaks caused by structural instability of the plastic bags, the formation of algal films on surfaces and subsequent fouling of the system, difficulties in cleaning the system diligently enough to avoid contamination, the potential presence of toxic elements in the plastic or within the carbon dioxide feed, and poor algal growth rates and low cell densities when $C$. reinhardtii cultures are grown under ambient photoautotrophic conditions. The key operational challenges will involve the development of $\mathrm{H}_{2}$ and biomass extraction systems, the establishment of an in situ technique to measure, control and recycle nutrients effectively, and the automation of the system through the ability to control the timing of the nutrient feed and biomass drain valves. Once these challenges have been overcome, the full semi-continuous PBR can be assembled to showcase the biophotolytic $\mathrm{H}_{2}$ production process at the demonstration scale.

\section{Conclusion}

Four key developments in biophotolytic $\mathrm{H}_{2}$ production by C. reinhardtii have been presented in this study: the design of a novel flat-plate PBR to facilitate green algal $\mathrm{H}_{2}$ production, ${ }^{20}$ the development of a nutrient control technique to enable spontaneous $\mathrm{H}_{2}$ production, ${ }^{19}$ the development of sulphur dilution and sulphur feed techniques to extend the $\mathrm{H}_{2}$ production process lifetime, and the conceptual design of a semi-continuous large-scale PBR. The novel flat-plate PBR proved effective at facilitating $C$. reinhardtii growth and $\mathrm{H}_{2}$ production at the laboratory scale, and $\mathrm{H}_{2}$ production was successfully and accurately measured by MIMS. Experiments in the flat-plate PBR enabled the development of a novel nutrient control technique that results in spontaneous $\mathrm{H}_{2}$ production following algal growth, achieving the objective for a continuous growth and $\mathrm{H}_{2}$ production cycle within a single PBR. $\mathrm{H}_{2}$ production by nutrient control was not only more practical and scalable than the alternative centrifugation process, it also led to superior $\mathrm{H}_{2}$ production rates and yields, which were $1.52 \mathrm{ml} \mathrm{H}_{2} \mathrm{l}^{-1} \mathrm{~h}^{-1}$ and $119.8 \mathrm{ml} \mathrm{H}_{2} \mathrm{l}^{-1}$ respectively. The dilution technique of sulphur re-insertion was used to develop a cyclic growth and $\mathrm{H}_{2}$ production process. A 95\% dilution resulted in near-complete algal culture recovery and a secondary $\mathrm{H}_{2}$ production stage with a $\mathrm{H}_{2}$ production rate of $1.13 \mathrm{ml} \mathrm{H}_{2} \mathrm{l}^{-1} \mathrm{~h}^{-1}$. The sulphur feed technique was used to operate the PBR as a chemostat for sulphate and maintain low sulphate concentrations during anaerobic $\mathrm{H}_{2}$ production. $\mathrm{H}_{2}$ production eventually failed despite the presence of sulphur in the algal medium, indicating that irreversible changes in algal metabolism under anaerobic conditions limit the effectiveness of the sulphur feed technique. The conceptual design of a semicontinuous PBR presents one possibility for practical and economical scale-up of biohydrogen production. The green algae $C$. reinhardtii are capable of producing a clean, efficient and sustainable biofuel through the biophotolysis of water to $\mathrm{H}_{2}$. Unfortunately, the $\mathrm{H}_{2}$ production rate remains very low, with a photochemical efficiency of less than one percent. The success of genetic engineering approaches aimed at improving C. reinhardtii light-harvesting, electron transport and $\mathrm{H}_{2}$ production efficiencies will eventually determine the commercial viability of the process. In the meantime, flexible engineering solutions, in the form of practical PBRs, measurement techniques and novel methodologies, are urgently required to facilitate present and future biophotolytic $\mathrm{H}_{2}$ production processes. This study makes a small but important step towards providing these engineering solutions and achieving the goal of sustainable $\mathrm{H}_{2}$ production.

\section{Nomenclature}

\section{C. reinhardtii}

Unicellular green alga Chlamydomonas reinhardtii

cc124 Laboratory wild-type strain of C. reinhardtii

Chl Chlorophyll

$\mathrm{H}_{2} \quad$ Molecular hydrogen

HPLC High-Performance Liquid Chromatography

IC Ion chromatography

LED Light-emitting diode

MIMS Membrane-Inlet Mass Spectrometry

OD Optical density

PBR Photobioreactor

PDMS Polydimethylsiloxane 
$\mathrm{pO}_{2} \quad$ Dissolved oxygen content

PSII Photosystem II reaction centre

TAP Tris-acetate phosphate algal growth medium

\section{Acknowledgements}

The authors would like to thank Paul Crudge for helping them to design and construct the flat-plate PBR, and Professor Peter J. Nixon for providing the $C$. reinhardtii cultures used in this study. The Solar Hydrogen project was funded by the UK Engineering and Physical Sciences Research Council (EPSRC), project reference EP/F00270X/1.

\section{References}

1 D. Stern, Stern review: the economics of climate change, 2006. 2 R. H. Wijffels and M. J. Barbosa, Science, 2010, 329, 796-799. 3 M. Ricci, P. Bellaby and R. Flynn, Energy Policy, 2010, 38, 2633-2640.

4 R. M. Navarro, M. C. Alvarez-Galván, J. A. Villoria de la Mano, S. M. Al-Zahrani and J. L. G. Fierro, Energy Environ. Sci., 2010, 3, 1865-1882.

5 J. Hansen, M. Sato, P. Kharecha, G. Russell, D. W. Lea and M. Siddall, Philos. Trans. R. Soc., A, 2007, 365, 1925-1954.

6 E. Stephens, I. L. Ross, J. H. Mussgnug, L. D. Wagner, M. A. Borowitzka, C. Posten, O. Kruse and B. Hankamer, Trends Plant Sci., 2010, 15, 554-564.

7 M. L. Ghirardi, A. Dubini, J. Yu and P.-C. Maness, Chem. Soc. Rev., 2009, 38, 52-61.

8 A. Melis, L. Zhang, M. Forestier, M. L. Ghirardi and M. Seibert, Plant Physiol., 2000, 122, 127-136.

9 S. J. Burgess, B. Tamburic, F. W. Zemichael, K. Hellgardt and P. J. Nixon, Adv. Appl. Microbiol., 2011, 75, 71-110.

10 A. Melis and T. Happe, Photosynth. Res, 2004, 80, 401-409.

11 S. N. Kosourov, A. A. Tsygankov, M. Seibert and M. L. Ghirardi, Biotechnol. Bioeng., 2002, 78, 731-740.

12 I. Akkerman, M. Janssen, J. Rocha and R. H. Wijffels, Int. J. Hydrogen Energy, 2002, 27, 1195-1208.

13 C. Posten and G. Schaub, J. Biotechnol., 2009, 142, 64-69.

14 C. U. Ugwu, H. Aoyagi and H. Uchiyama, Bioresour. Technol., 2008, 99, 4021-4028.
15 D. S. Gorman and R. P. Levine, Plant Physiol., 1966, 41, 1643-1647.

16 A. A. Tsygankov, S. N. Kosourov, I. V. Tolstygina, M. L. Ghirardi and M. Seibert, Int. J. Hydrogen Energy, 2006, 31, 1574-1584.

17 B. Degrenne, J. Pruvost, G. Christophe, J. F. Cornet, G. Cogne and J. Legrand, Int. J. Hydrogen Energy, 2010, 35, 10741-10749.

18 T. V. Laurinavichene, I. V. Tolstygina and A. A. Tsygankov, J. Biotechnol., 2004, 114, 143-151.

19 B. Tamburic, F. W. Zemichael, G. C. Maitland and K. Hellgardt, Int. J. Hydrogen Energy, 2012, 37, 8988-9001.

20 B. Tamburic, F. W. Zemichael, P. Crudge, G. C. Maitland and K. Hellgardt, Int. J. Hydrogen Energy, 2011, 36, 6578-6591.

21 D. I. Arnon, Plant Physiol., 1949, 24, 1-15.

22 M. L. Ghirardi, L. Zhang, J. W. Lee, T. Flynn, M. Seibert, E. Greenbaum and A. Melis, Trends Biotechnol., 2000, 18, 506-511.

23 P. C. Hallenbeck and J. R. Benemann, Int. J. Hydrogen Energy, 2002, 27, 1185-1193.

24 M.-Y. Ding, H. Koizumi and Y. Suzuki, Anal. Sci., 1995, 11, 239-243.

25 M. Janssen, J. Tramper, L. R. Mur and R. H. Wijffels, Biotechnol. Bioeng., 2003, 81, 193-210.

26 K. Beckmann, J. Messinger, M. R. Badger, T. Wydrzynski and W. Hillier, Photosynth. Res., 2009, 102, 511-522.

27 B. Hankamer, F. Lehr, J. Rupprecht, J. H. Mussgnug, C. Posten and O. Kruse, Physiol. Plant., 2007, 131, 10-21.

28 J. P. Kim, K.-R. Kim, S. P. Choi, S. J. Han, M.-S. Kim and S. J. Sim, Int. J. Hydrogen Energy, 2010, 35, 13387-13391.

29 N. Rossignol, L. Vandanjon, P. Jaouen and F. Que, Aquacult. Eng., 1999, 20, 191-208.

30 J. Sheehan, T. Dunahay, J. R. Benemann and P. Roessler, A look back at the US Department of Energy's Aquatic Species Program: Biodiesel from algae, 1998.

31 H. K. Webb, J. Arnott, R. J. Crawford and E. P. Ivanova, Polymers, 2013, 5, 1-18.

32 L. K. Massey, Permeability properties of plastics and elastometers, Plastic Design Library, New York, 2003.

33 K. Toi, K. Takeuchi and T. Tokuda, J. Polym. Sci., 1980, 18, 189-198. 